\title{
Answer to the comments by Eric J. Grant et al. in "Radiation unlikely to be responsible for high cancer rates among distal Hiroshima A-bomb survivors"
}

\author{
Masaru Miyao - Tomoyuki Watanabe · \\ Ryumon Honda $\cdot$ Yuichi Yamada
}

Received: 31 March 2009/Accepted: 8 April 2009/Published online: 2 May 2009

(C) The Japanese Society for Hygiene 2009

Eric J. Grant et al. raised three issues concerning our paper "Hiroshima survivors exposed to very low doses of A-bomb primary radiation showed a high risk for cancers" [1]. Their proposed title was "Radiation unlikely responsible for high cancer rates among distal Hiroshima A-bomb survivors". However, in response, we suggest that a better title would be "Risk analysis in LSS without using an unexposed group is responsible for the apparent low cancer rates among distal Hiroshima A-bomb survivors". We discuss the reasons for this proposal here.

The reasons for the differences in sex-specific risks among distal Hiroshima A-bomb survivors

Grant et al. wrote "the standardized mortality ratio (SMR) values [1] for VLD (the 'very low dose' category: 0-0.005 Sv, 2.5-10 km from the hypocenter) women were very close to 1.0 ; only those for men were elevated. In a related observation, previous studies have shown that the excess relative risk (ERR) per gray (Gy) of cancer mortality after radiation exposure is consistently higher for

M. Miyao ( $\square)$

Graduate School of Information Science,

Nagoya University, Furo-cho,

Chikusa-ku, Nagoya 464-8601, Japan

e-mail: mmiyao@med.nagoya-u.ac.jp

T. Watanabe

Department of Health and Nutrition,

Faculty of Psychological and Physical Science,

Aichi Gakuin University, Nisshin, Japan

R. Honda · Y. Yamada

Department of Social and Environmental Medicine (Hygiene),

Kanazawa Medical University, Ishikawa, Japan women compared to men $[2,3]$ ”. However, references [2, 3] cited by Grant et al. were both studies of primary radiation only; they did not consider fallout or residual radiation. Our study was carried out taking the latter into account, and we eventually came to the conclusion that it was very possible that the high cancer mortality risk among the VLD group was affected by residual radiation.

A series of Life Span Studies (LSS) by the Radiation Effects Research Foundation (RERF) estimated the "risk of the unexposed group" by extrapolating the results for the exposed group using a "sophisticated" statistical analysis-Poisson regression analysis-without using a genuine unexposed group. Pierce wrote "the LSS cohort includes most survivors within about $2.5 \mathrm{~km}$ of the bombings who lived in Hiroshima or Nagasaki in 1950 and who met certain conditions ensuring adequate follow-up. It includes a comparison group of comparable size, matched by sex and age, selected from survivors who were within $2.5-10 \mathrm{~km}$ of the hypocenter, where radiation exposures were low or negligible" [2]. The risk for cancers in the VLD subjects was revealed to be fairly high when compared to the population of Hiroshima or Okayama prefectures in our research. However, RERF calculated the background risk using this high risk in the VLD subjects.

In the commentary, Grant et al. calculated the sex ratio of ERR for solid cancer using our research results and concluded, "this sex ratio of excess risks (female/ male) is greater than unity in the high dose (HD) group as expected, but is less than unity for the low dose (LD) group and even smaller for the VLD group. This pattern supports the conclusion that the dominant cause for the high solid cancer SMR observed in the HD group is radiation, whereas, in the LD and VLD groups, the elevated SMRs are primarily due to non-radiation factors". 
What is indicated as a result from the LSS studies of RERF (references [2, 3]) is that, taking the VLD group in our study as a 0 dose control, replication can be done easily if the risks of the LD and HD groups are divided by the risk of the VLD group (Table 1). This may be seen as a simulation of the above-mentioned ERR calculation, taking exposure victims as controls. Among men, the risk of the VLD group was high against that of a "genuine nonexposed standard group". The risk ratios in the LD and HD categories against those individuals they call controls (made with calculations from the exposure group that we classified as VLD) are also low. In women in the VLD group, however, the risk is nearly the same (SMR is about 1.0 ), and in women in the other dose classifications (LD and HD) the relative risks are not much different than the RERF results. In fact, as shown in the upper part of table (comparing Hiroshima prefecture), taking the SMR of VLD in our analysis results as a standard (1.0) and calculating the SMR ratio for $\mathrm{LD}$ and $\mathrm{HD}$, the risk ratios for men were 1.015 and 1.190 in the LD and HD groups, respectively, and those for women were 1.061 and 1.588 in the LD and HD groups respectively. In the lower part of table (comparing Okayama prefecture), the risk ratios were 1.011 (LD) and 1.190 (HD) for men and 1.063 (LD) and 1.591 (HD) for women. Thus, when the SMRs of the VLD are considered to be the standard group, using the estimates from the upper part of table (Hiroshima), the sex ratio of the new "excess risk ratio (risk ratio: 1)" for solid cancer is greater than unity in both the HD group (female/ male $=0.588 / 0.190=3.10)$ and the $\operatorname{LD}$ group $(0.061 /$ $0.015=4.07)$, as expected. Grant et al. seem happy to see that the "ERR" (standard: SMR of the VLD) of cancer mortality after radiation exposure is consistently higher for women than for men. Furthermore, as expected, in the series of LSS by RERF, the risk in men is estimated to be lower than the risk we calculated, and the risk in women appears to be higher than that in men. This is no more than a trick to unduly and artificially lower the ERR with Poisson regression analysis within the exposed group, with subjects more than $2.5 \mathrm{~km}$ away (VLD) used as the standard group.

Thus, the difference between the results of our study and those reported in references [2, 3] are due to differences in study design. Our study, which used a control group, can be used to assess the validity of those studies, but it is difficult to use the studies reported in references [2, 3], which did not use a control group, to assess the validity of ours.

In our analysis, the risk of males in the VLD group (standard: Hiroshima and Okayama prefectures matching follow-up interval, sex, and age in 1945) tended to be higher than that in females, but the cause of this can be reasonably accounted for by the fact that men spent more time outdoors in relief work and other activities. This is a critical factor when investigating the SMR of men and women who were exposed in distal areas.

The evidence on which the assertion of Grant et al. relies was derived from the fatal flaw in their study design of using exposure victims (VLD) as controls. Therefore, arguments based on figures resting on such a foundation are implausible.

\section{Plausibility of the effects among distal area}

The reasons that the effects of residual radiation in Hiroshima prefecture have been ignored or discounted include (1) there were no direct measurements of particulate radioactivity in the fallout immediately after the explosion, and (2) although there are measurement data for radioactive substances (cesium 137) in the soil, including that contained in radioactive rain collected during the 3 days following the explosion in Hiroshima [4], the values in other data were low, with the exception of the Koi and Takasu area, as pointed out by Grant et al. However, nearly all subsequent measurements of residual radiation were made following the Makurazaki typhoon on 17 September 1945 and a subsequent typhoon on 9 October. The possibility that these values were low because much of the radioactive substances in the soil had already been washed away cannot be ruled out. Therefore, even limiting radioactive fallout to radioactive rain, measurements of residual radioactivity are unreliable and cannot be stated with certainty. On this point, even the DS86 stated as follows: "Many factors affecting the accuracy of the measurements are not well known 40 years after the bombs, therefore exposure estimates must be rough approximations. In general, the exposure rates were not measured soon enough to avoid some weathering and they were not repeated often enough to account for subsequent weathering or to provide a time distribution of radioactivity. The number of sites monitored was too small to develop a good estimate of the detailed geographic distribution of the radioactivity. Also, in such surveys, it is difficult to avoid unrepresentative sampling and it is not known whether such a sampling bias exists. Finally, the details of calibration and measurement are not always available [5]". There is no mention in DS02 of these residual radioactivity measurements. DS86 and DS02 define the dose of radiation as the primary radiation only. The possibility cannot be refuted, therefore, that the VLD group was subjected to fairly high exposure through fallout and residual radiation.

In the process of calculating the SMR, the data on observed number of deaths that we used in the numerator were data published by RERF. The figures for the expected number of deaths used in the denominator were calculated from data published in vital statistics. The SMR ratio 


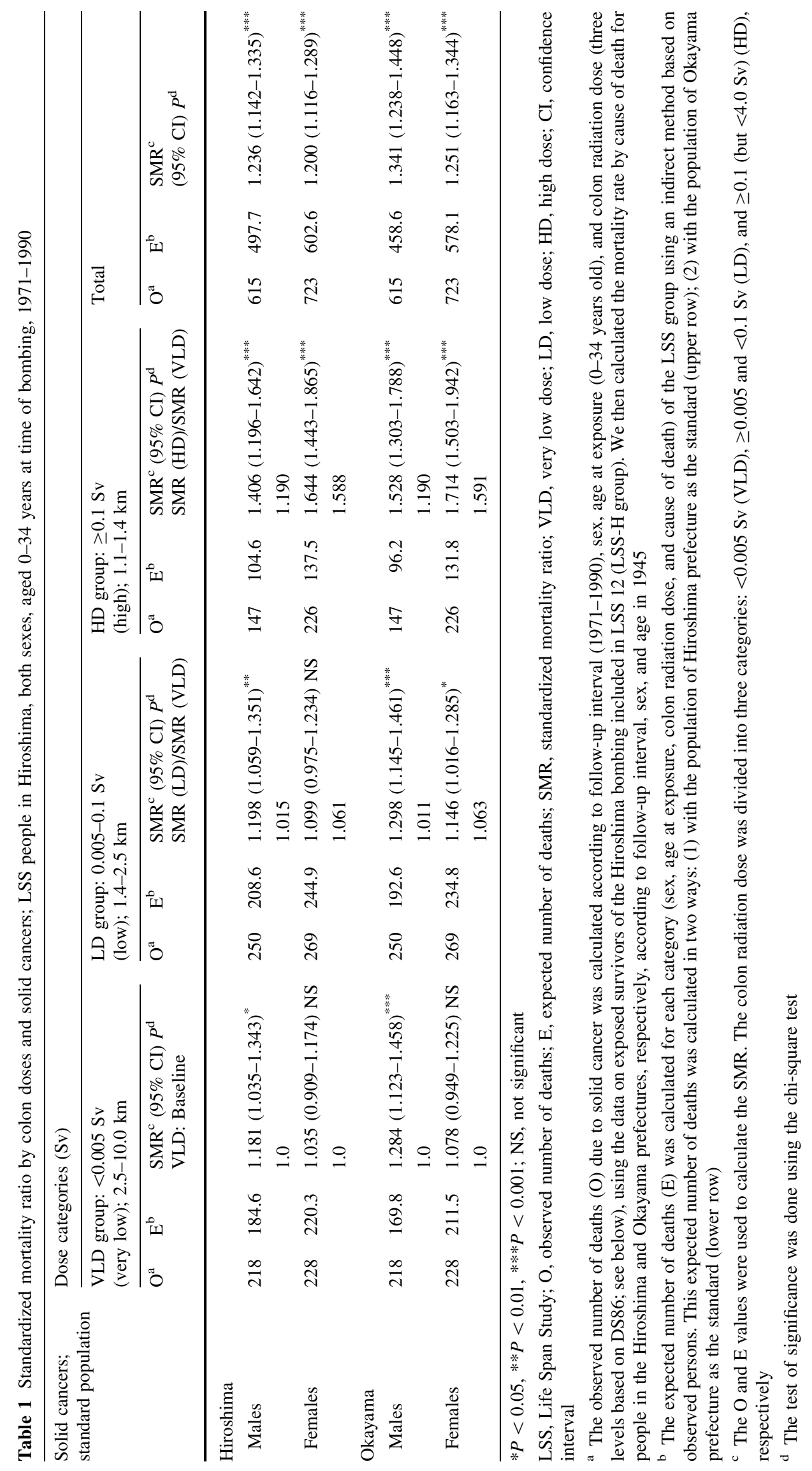


(relatively large difference) for the different dosage classifications was derived from public data from RERF and the Japanese government that have been collected since 1971, and we did not have any opportunity to be selective in our use of these data.

\section{Other points}

Grant et al. wrote, "WMHY [1] suggest that (1) underestimated initial doses and/or (2) higher than assumed sensitivities to initial exposures could be responsible for the VLD group's high SMRs". However, we would like to emphasize that we were only enumerating possibilities based on the logical context. We suggested nothing more than that these are two theoretically possible causes for the elevated SMR in VLD. In fact, compared with the studies of RERF, which did not establish a genuine non-exposed control group, we demonstrated that the SMR in VLD was rather high by adopting the residents of both Hiroshima and Okayama prefectures as a standard population, with matching for sex, age, and observation period.

Grant et al. stated that "DS02 includes improvements in the source terms, radiation transport and shielding assessments, and has been validated by the results of extensive physical measurements", and "an argument based on higher sensitivities to low initial doses also seems to be unsupported as neither the RERF data [6], nor the weight of evidence from other radiation studies [7, 8] show higher risks per unit dose at low doses than at higher doses". If these statements are true, the hypotheses of the (1) underestimated initial doses and (2) the possibility that higher than assumed sensitivities to initial exposures are responsible for the VLD group's high SMRs should be denied. Therefore, the high SMR of people exposed to very low doses suggests the possibility that the effects of fairly high residual radiation exist in those exposed in distal areas of more than $2.5 \mathrm{~km}$ from the hypocenter $(<0.005 \mathrm{~Sv}$ with the definition in DS86).

Grant et al. also described the problems that can occur with the DS86 dose estimation system and the new DS02 dose estimation system. However, the questions of just what was considered in the process of revising DS02 from DS86 and whether these items were satisfactorily dealt with is not relevant to the issues in our study. In any event,
DS02 is a system of estimating doses of radiation in the initial period and does not describe residual radiation; consequently it cannot be said that there is no effect from residual radiation on the basis of DS02.

\section{Conclusions}

We are grateful to RERF for making these data available and enabling valuable risk analysis. However, the exposure risk cannot be accurately calculated with these published data alone. Conducting a risk analysis without a genuine non-exposed group does not do justice to these valuable data. We would like to recommend that RERF look at new research results with an open mind and change their fundamental research model by establishing a non-exposed control group.

\section{References}

1. Watanabe T, Miyao M, Honda R, Yamada Y. Hiroshima survivors exposed to very low doses of A-bomb primary radiation showed a high risk for cancers. Environ Health Prev Med. 2008;13:264-70.

2. Pierce DA, Shimizu Y, Preston DL, Vaeth M, Mabuchi K. Studies of the mortality of atomic bomb survivors. Report 12, Part I. Cancer: 1950-1990. Radiat Res. 1996;146:1-27.

3. Preston DL, Pierce DA, Shimizu Y, Cullings HM, Fujita S, Funamoto $S$, et al. Effect of recent changes in atomic bomb survivor dosimetry on cancer mortality risk estimates. Radiat Res. 2004;162:377-89.

4. Shizuma K, Iwatani K, Hasai H, Hoshi M, Oka T, Okano M. ${ }^{137} \mathrm{Cs}$ concentration in soil samples from an early survey of Hiroshima atomic bomb and cumulative dose estimation from the fallout. Health Phys. 1996;71:340-6.

5. Okajima S, Fujita S, Harley H. Radiation doses from residual radioactivity. In: Roesch WC (ed) US-Japan joint reassessment of atomic bomb radiation dosimetry in Hiroshima and Nagasaki, final report, vol 1 (pp 205-226). Hiroshima: Radiation Effects Research Foundation; 1987.

6. Ban S, Cologne JB, Fujita S, Awa AA. Radiosensitivity of atomic bomb survivors as determined with a micronucleus assay. Radiat Res. 1993;134:170-8.

7. Effects of Ionizing Radiation: UNSCEAR 2006 Report to the General Assembly, with scientific annexes/United Nations Scientific Committee on the Effects of Atomic Radiation (UNSCEAR). New York: United Nations; 2008.

8. National Research Council. Committee to assess health risks from exposure to low levels of ionizing radiation (BEIR VII). Washington D.C.: National Academic Press; 2005. 